\title{
A moving grid finite element method applied to a model biological pattern generator is
}

\author{
Anotida Madzvamuse ${ }^{\mathrm{a}, *}$, Andrew J. Wathen ${ }^{\mathrm{b}}$, Philip K. Maini ${ }^{\mathrm{c}}$ \\ a Mathematics Department, 221 Parker Hall, Auburn University, AL 36849-3510, USA \\ ${ }^{\mathrm{b}}$ Numerical Analysis Group, Oxford University Computing Laboratory, Wolfson Building, Parks Road, Oxford OX1 3QD, UK \\ ' Centre for Mathematical Biology, Mathematical Institute, University of Oxford, 24-29 St. Giles', Oxford OX1 3LB, UK
}

Received 2 September 2002; received in revised form 10 April 2003; accepted 26 May 2003

\begin{abstract}
Many problems in biology involve growth. In numerical simulations it can therefore be very convenient to employ a moving computational grid on a continuously deforming domain. In this paper we present a novel application of the moving grid finite element method to compute solutions of reaction-diffusion systems in two-dimensional continuously deforming Euclidean domains. A numerical software package has been developed as a result of this research that is capable of solving generalised Turing models for morphogenesis.
\end{abstract}

(c) 2003 Elsevier B.V. All rights reserved.

Keywords: Moving grid finite elements; Reaction-diffusion systems; Turing instability; Colour pattern formation; Butterfly wing; Papilio dardanus

\section{Introduction}

Understanding how spatial pattern arises is a central but still unresolved issue in developmental biology. It is clear that genes play a crucial role in embryology but the study of genetics alone can not explain how the complex mechanical and chemical spatio-temporal signalling cues which determine cell fate are set up and regulated in the early embryo. These signals are a consequence of many nonlinear interactions and mathematical modelling and numerical computation have an important role to play in understanding and predicting the outcome of such complex interactions.

The application of mathematical modelling to problems in developmental biology has given rise to a variety of models which account for spatio-temporal patterning phenomena. In the chemical prepattern model approach it is hypothesised that a spatially homogeneous or uniform density of cells responds to some spatially heterogeneous chemical pattern and differentiates according to some rules. Turing [49] in his

\footnotetext{
This work supported by EPSRC Life Sciences Initiative Grant GR/R03914.

* Corresponding author. Fax: +1-334-844-6555.

E-mail address: anotida.madzvamuse@comlab.ox.ac.uk (A. Madzvamuse).
} 
seminal paper concerning morphogenesis, suggested a novel concept of diffusion driven instability in reaction-diffusion systems. Since then, the reaction-diffusion theory of pattern formation has been widely applied to biology, ecology [42], semiconductor physics [6], material science [23], hydrodynamics [50], astrophysics [35], chemistry and economics. Typical examples of biological applications of reaction-diffusion systems are: pattern formation in hydra [19], animal coat markings [32], butterfly wing pigmentation patterns [34], skeletal patterning in limb development (see for review [27]) and shell pigmentation patterns [28]. Detailed studies of these models have been carried out on spatial domains of one dimension and two dimensions with simple geometries. In nearly all these cases, the domain was considered fixed in size, however recent experimental evidence shows that domain growth may play a crucial role in pattern formation [22].

From the biologically realistic assumption that growth occurs via a continuous deformation of the domain, we can solve the system numerically using the finite element method on a continuously deforming domain [4]. The essential component underlying this method is the representation of the object function by a piecewise linear continuous function on a finite element mesh in which the nodal positions vary with time. The way in which these nodes are moved and how the solution evolves is derived in the weak form of the model system. The nodal movement can either be provided by a separate definition or by a mechanism: we will prescribe a specific definition for the motion of the domain and correspondingly define the motion of all the nodes in the grid. This is the key difference to the moving finite element method invented by Miller [29]. Miller does not specify the motion of the nodes, instead the nodal movement is generated automatically by minimising the residual of the differential equation. In his method mesh tangling may result whereas this can be avoided with prescribed mesh motion (see [12,13] for more details on mesh tangling and other problems). Although our work will be mainly on a two-dimensional moving grid, Sun W. [46], use the moving mesh method to solve the one-dimensional Gierer-Meinhardt model.

In Section 2 we present the model equations considered in this paper. The moving grid finite element theory is described in Section 3. Here we derive the weak form of the partial differential equation and describe the Galerkin finite element approximation on a continuously deforming grid. Numerical experiments are presented in Section 4 on regular and irregular growing domains. Lastly in Section 5 we present conclusions and discuss the implications of the novel application of the moving finite element method to pattern formation and transition in biological problems. We also provide numerical software and documentation for the methods described in this paper which can be freely downloaded from http://web.comlab.ox.ac.uk/oucl/work/andy.wathen/software.html.

\section{Model equations}

The numerical experiments in this paper will be restricted to a generalised reaction-diffusion system of two chemical species which can be written in terms of non-dimensional variables as

$$
\frac{\partial \mathbf{u}}{\partial t}=\gamma \mathbf{f}(\mathbf{u})+\mathbf{P}_{3}(\mathbf{u})+\mathbf{D} \nabla^{2} \mathbf{u} \quad \text { in } \Omega(t)
$$

with $\Omega(t)$ representing a time-dependent domain. Here we define

$$
\mathbf{u}=\left(\begin{array}{l}
u \\
v
\end{array}\right), \quad \mathbf{f}=\left(\begin{array}{l}
f(u, v) \\
g(u, v)
\end{array}\right), \quad \mathbf{P}_{\mathbf{3}}=\left(\begin{array}{l}
p_{3}(u, v) \\
q_{3}(u, v)
\end{array}\right), \quad \mathbf{D}=\left(\begin{array}{cc}
D_{u} & 0 \\
0 & D_{v}
\end{array}\right), \quad \mathbf{x}=(x(t), y(t)),
$$

where $u, v$ are the two chemical concentrations under investigation, $f, g, p_{3}, q_{3}$ are reaction kinetics, and $\mathbf{D}$ is the diffusion matrix ( $D_{u}$ and $D_{v}$ are constant diffusion parameters). In a number of important applications the parameters $\gamma$ and $d$ are the most important and this is why they are explicitly included in (2.1). 
Initial conditions are given by $\mathbf{u}(\mathbf{x}, 0)=\mathbf{u}_{0}(\mathbf{x})$ where $\mathbf{u}_{0}(\mathbf{x})$ is a prescribed non-negative continuous bounded function. Boundary conditions can be of Dirichlet type ( $u$ and/or $v$ given on the boundary) or of (homogeneous) Neumann type which describe zero-flux of $u$ (or $v$ ) out of the boundary. Initial conditions are defined as small random perturbations about the uniform homogeneous steady state, if it exists (i.e., a state $u=$ constant, $v=$ constant satisfying the boundary conditions and equations (2.1)).

Below we consider three special cases where $\gamma \neq 0$ and $p_{3}(u, v)=0=q_{3}(u, v)$. In each case we give a brief description of each model and its physical interpretation.

\subsection{Gierer-Meinhardt reaction kinetics}

This is a phenomenological model suggested by Gierer and Meinhardt [19] whereby reaction kinetics are chosen in such a way that one of the chemicals (termed activator) activates the production of the other chemical (the inhibitor) which, in turn, inhibits the production of the activator. The non-dimensionalised reaction-diffusion system is given by

$$
\begin{aligned}
& \frac{\partial u}{\partial t}=\gamma\left(a-b u+\frac{u^{2}}{v\left(1+k u^{2}\right)}\right)+\nabla^{2} u, \\
& \frac{\partial v}{\partial t}=\gamma\left(u^{2}-v\right)+\mathrm{d} \nabla^{2} v
\end{aligned}
$$

where $u(x, y, t)$ is the concentration of the activator, $v(x, y, t)$ is the concentration of the inhibitor, $t$ is time and $\nabla^{2}=\left(\partial^{2} / \partial x^{2}\right)+\left(\partial^{2} / \partial y^{2}\right)$ is the two-dimensional Laplacian. $a, b, d$ and $\gamma$ are all non-dimensionalised positive parameters and $k$ is a measure of the saturation concentration (see for example [33]). The biological interpretation of the reaction kinetics in (2.2) is that $u$ is produced at a constant rate $\gamma a$ and is degraded linearly at rate $\gamma b$. The $\gamma\left[u^{2} / v\left(1+k u^{2}\right)\right]$ term implies autocatalysis in $u$ with saturation at high concentration values of $u$, and inhibition of $u$ through the production of $v$. In Eq. (2.3), $v$ is activated (produced) by $u$ and degraded linearly.

\subsection{Thomas reaction kinetics}

This model is based on a specific substrate-inhibition reaction involving the substrates oxygen $(v(x, y, t))$ and uric acid $(u(x, y, t))$ which react in the presence of the enzyme uricase. The reaction kinetics, derived by fitting the kinetics to experimental data [47], can be written in non-dimensional form as

$$
\begin{aligned}
& \frac{\partial u}{\partial t}=\gamma(a-u-h(u, v))+\nabla^{2} u, \\
& \frac{\partial v}{\partial t}=\gamma(\alpha b-\alpha v-h(u, v))+\mathrm{d} \nabla^{2} v
\end{aligned}
$$

with $h(u, v)=\rho u v /\left(1+u+K u^{2}\right)$. Here $a, b, d, \gamma, \alpha, \rho$ and $K$ are positive parameters. The term $h(u, v)$ indicates the rate at which $u$ and $v$ are used up, in particular $h(u, v)$ exhibits what is known as substrateinhibition, that is, for small $u, h(u, v)$ increases with $u$, while it decreases with large $u$.

\subsection{Schnakenberg reaction kinetics}

This is one of the simplest reaction-diffusion models. It is derived from a series of hypothetical trimolecular autocatalytic reactions proposed by Schnakenberg [41]. In non-dimensional form the system is written as 


$$
\begin{aligned}
& \frac{\partial u}{\partial t}=\gamma\left(a-u+u^{2} v\right)+\nabla^{2} u, \\
& \frac{\partial v}{\partial t}=\gamma\left(b-u^{2} v\right)+\mathrm{d} \nabla^{2} v,
\end{aligned}
$$

where $a, b, d$ and $\gamma$ are positive parameters. The biological interpretation follows as above, with the term $u^{2} v$ representing nonlinear activation of $u$ and nonlinear consumption for $v$.

\subsection{Other reaction kinetics}

If $\gamma=0, p_{3}(u, v) \neq 0$ and $q_{3}(u, v) \neq 0$ then the reaction-diffusion system (2.1) becomes

$$
\frac{\partial \mathbf{u}}{\partial t}=\mathbf{P}_{3}(\mathbf{u})+\mathbf{D} \nabla^{2} \mathbf{u}
$$

where

$$
\begin{aligned}
& p_{3}(u, v)=a_{0}+a_{1} u+a_{2} v+a_{3} u^{2}+a_{4} u v+a_{5} v^{2}+a_{6} u^{3}+a_{7} u^{2} v+a_{8} u v^{2}+a_{9} v^{3}, \\
& q_{3}(u, v)=b_{0}+b_{1} u+b_{2} v+b_{3} u^{2}+b_{4} u v+b_{5} v^{2}+b_{6} u^{3}+b_{7} u^{2} v+b_{8} u v^{2}+b_{9} v^{3}
\end{aligned}
$$

are general bivariate cubic polynomials with $a_{i}$ and $b_{i}(i=0, \ldots, 9)$ being constant coefficients. Note that the Schnakenberg reaction kinetics is a special case of (2.8). Another special case is the Gray and Scott $[17,18]$ model. More special cases can be found in the papers by Barrio et al. [7,8] and Aragón et al. [2,3].

\subsection{Parameter values}

The parameter values in the reaction terms are those that give a constant uniform steady state in the absence of diffusion. In our investigations, these are taken from the literature [33]. However, the values of $\gamma$ and the diffusion coefficient $d$ are determined from the conditions that give rise to Turing instability. Turing instability or diffusion-driven instability occurs when a uniform steady state, linearly stable in the absence of diffusion, goes unstable in the presence of diffusion. The process of determining these parameter values is known as linear stability analysis (Appendix A). On regular two-dimensional shapes, standard linear stability analysis yields general solutions which are linear combinations of products of sine or cosine functions with mode $(m, n)$. These are the eigenfunctions of the Laplacian with homogeneous Neumann or Dirichlet (at the uniform steady state) boundary conditions. Comparisons will be made between the numerically computed inhomogeneous steady-state solutions and their corresponding eigenfuctions on regular domains.

\section{The moving grid finite element method}

Let $\mathbf{U}$ be the finite element approximation to $\mathbf{u}$ defined as

$$
\mathbf{U}(\mathbf{x}, t)=\sum_{i=0}^{N+1} \mathbf{U}_{i}(t) \phi_{i}(\mathbf{x}, \mathbf{a}(t)),
$$

where $\mathbf{x} \in \mathbb{R}^{m}$ indicates the spatial coordinates and $\mathbf{a}(t)$ represents the grid in time, that is, the vector of nodal positions at time $t$. This grid is given by $\mathbf{a}(t)=\left(a_{0}(t), a_{1}(t), \ldots, a_{N+1}(t)\right)^{\mathrm{T}}$ where $\mathbf{a}_{i}(t)=\left(x_{i}(t), y_{i}(t)\right)^{\mathrm{T}}$ is the position vector of the $i$ th node. Here, $\phi_{i}(\mathbf{x}, \mathbf{a}(t))$ is the piecewise linear hat function, which is defined to 
be the unique piecewise linear and continuous function on the grid such that $\phi_{i}\left(\mathbf{a}_{j}, \mathbf{a}(t)\right)=\delta_{i, j}$. We calculate the time derivative of the finite element approximation $\mathbf{U}$ as given by

$$
\frac{\partial \mathbf{U}}{\partial t}=\sum_{i=0}^{N+1} \frac{\partial}{\partial t}\left(\mathbf{U}_{i}(t) \phi_{i}(\mathbf{x}, \mathbf{a}(t))\right) .
$$

Applying the product rule we have

$$
\frac{\partial \mathbf{U}}{\partial t}=\sum_{i=0}^{N+1}\left[\frac{\mathrm{d} \mathbf{U}_{i}}{\mathrm{~d} t} \phi_{i}+\mathbf{U}_{i} \frac{\partial \phi_{i}}{\partial t}\right] .
$$

Now applying the chain rule to the last term we obtain [20]

$$
\frac{\partial \mathbf{U}}{\partial t}=\sum_{i=0}^{N+1} \frac{\mathrm{d} \mathbf{U}_{i}}{\mathrm{~d} t} \phi_{i}+\sum_{i=0}^{N+1} \beta_{i}^{\mathrm{T}} \dot{\mathbf{a}}_{i}(t),
$$

where $\beta_{i}=\partial \mathbf{U} / \partial \mathbf{a}_{i}$. Baines and Wathen [5] proved that $\beta_{i}=\partial \mathbf{U} / \partial a_{i}=-\phi_{i} \partial \mathbf{U} / \partial \mathbf{x}$ in multi-dimensions. In particular $\beta_{i}=-\phi_{i} \mathbf{U}_{x}$ with $\dot{\mathbf{a}}_{i}=\dot{x}_{i}$ in one dimension and $\beta_{i}=-\phi_{i}\left(U_{x} U_{y}\right)$ with $\dot{\mathbf{a}}_{i}=\left(\dot{x}_{i}, \dot{y}_{i}\right)^{\mathrm{T}}$ in two dimensions. Therefore the time derivative of $\mathbf{U}$ is given by

$$
\frac{\partial \mathbf{U}}{\partial t}=\sum_{i=0}^{N+1}\left[\dot{\mathbf{U}}_{i}-\dot{x}_{i} \mathbf{U}_{x}\right] \phi_{i}(\mathbf{x}, \mathbf{a}(t))
$$

in one dimension and in two dimensions by

$$
\frac{\partial \mathbf{U}}{\partial t}=\sum_{i=0}^{N+1}\left[\dot{\mathbf{U}}_{i}-\left(\dot{x}_{i} \mathbf{U}_{x}+\dot{y}_{i} \mathbf{U}_{y}\right)\right] \phi_{i}(\mathbf{x}, \mathbf{a}(t)) .
$$

It must be observed that Carlson and Miller [12,13] presented a simpler derivation for the time-derivative of $\mathbf{U}$ for triangles (and tetrahedrons, etc.) by considering a natural barycentric parametrisation involving the hat functions.

The effect of the growing domain is to add extra terms to the finite element formulation as illustrated in the general case (3.4) and in particular for (3.5) and (3.6) in the one- and two-dimensional cases, respectively. The key difference to the moving finite element method invented by Miller [30] is that we prescribe a specific definition for the nodal movement $\dot{\mathbf{a}}_{i}$. We assume that nodal movement can be determined or prescribed, for example, by growth rates derived from biological observations. This is the novelty of our numerical method. As far as we know this is the first work to demonstrate a novel way of moving the grid by use of a defined function which describes growth of the problem domain.

\subsection{Spatial discretisation: finite element method}

In order to discretise the model Eq. (2.1) in space, one has to divide the domain under consideration into a series of non-overlapping sub-domains or elements. The problem is then approximated within each of these elements, and the corresponding element contributions are summed to create a global system of equations.

The discretisation of the geometry is carried out by using triangular elements with a piecewise linear approximation to the solution. We impose the conditions that the elements must cover the entire domain without leaving gaps (except perhaps at the boundary), that is, any pair of elements intersect along a complete edge, at a vertex or not at all. If we cover a region $\Omega(t)$ with triangular elements, then each element 
can be mapped onto a standard fixed regular triangle at time $t$. This simplifies the required integration over each triangular element.

Let $\mathbf{U}$ be defined as in (3.1) such that the family of the functions $\phi_{i}$ are linearly independent. Although we consider a finite-dimensional space in practice for the basis functions $\phi_{i}$, we assume that as $N$ tends to infinity, convergence is possible since the limit space is dense in $H^{1}(\Omega(t))$. Over each triangular element, (e) say, $\mathbf{U}$ can be expressed as $\mathbf{U}^{(e)}(x, y)=c_{1}(t)+c_{2}(t) x(t)+c_{3}(t) y(t)$. We require that $\mathbf{U}^{(e)}\left(x_{i}, y_{i}, t\right)=\mathbf{U}_{i}^{(e)}$ $(i=1,2,3)$. Therefore

$$
\begin{aligned}
& \mathbf{U}_{1}(t)=c_{1}(t)+c_{2}(t) x_{1}(t)+c_{3}(t) y_{1}(t), \\
& \mathbf{U}_{2}(t)=c_{1}(t)+c_{2}(t) x_{2}(t)+c_{3}(t) y_{2}(t), \\
& \mathbf{U}_{3}(t)=c_{1}(t)+c_{2}(t) x_{3}(t)+c_{3}(t) y_{3}(t) .
\end{aligned}
$$

Solving this system of algebraic equations results in

$$
\begin{aligned}
& c_{1}(t)=\frac{1}{2 A_{123}(t)}\left(\alpha_{1} \mathbf{U}_{1}(t)+\alpha_{2} \mathbf{U}_{2}(t)+\alpha_{3} \mathbf{U}_{3}(t)\right), \\
& c_{2}(t)=\frac{1}{2 A_{123}(t)}\left(\beta_{1} \mathbf{U}_{1}(t)+\beta_{2} \mathbf{U}_{2}(t)+\beta_{3} \mathbf{U}_{3}(t)\right), \\
& c_{3}(t)=\frac{1}{2 A_{123}(t)}\left(\tau_{1} \mathbf{U}_{1}(t)+\tau_{2} \mathbf{U}_{2}(t)+\tau_{3} \mathbf{U}_{3}(t)\right),
\end{aligned}
$$

where $A_{123}(t)$ is the area of the triangle and $\alpha_{i}=x_{j}(t) y_{k}(t)-x_{k}(t) y_{j}(t), \quad \beta_{i}=y_{j}(t)-y_{k}(t)$, and $\tau_{i}=$ $-\left(x_{j}(t)-x_{k}(t)\right)$ where $i, j, k=1,2,3$. Substituting (3.10)-(3.12) into $\mathbf{U}^{(e)}$ results in $\mathbf{U}^{(e)}(x, y, t)=$ $\sum_{j=1}^{3} \mathbf{U}_{j}(t) \phi_{j}(x(t), y(t))$ where

$$
\begin{aligned}
& \phi_{1}=\frac{1}{2 A_{123}(t)}\left(\alpha_{1}+\beta_{1} x(t)+\tau_{1} y(t)\right), \\
& \phi_{2}=\frac{1}{2 A_{123}(t)}\left(\alpha_{2}+\beta_{2} x(t)+\tau_{2} y(t)\right), \\
& \phi_{3}=\frac{1}{2 A_{123}(t)}\left(\alpha_{3}+\beta_{3} x(t)+\tau_{3} y(t)\right) .
\end{aligned}
$$

Let each triangle be mapped onto a unit right-angled triangle by an affine transformation from global Cartesian coordinates $\mathbf{r}=(x(t), y(t))$ to local coordinates $(\xi, \eta)$ by linear hat functions defined by

$$
\mathbf{r}=(x(\xi, \eta, t), y(\xi, \eta, t))=(1-\xi-\eta)\left(x_{1}(t), y_{1}(t)\right)+\xi\left(x_{2}(t), y_{2}(t)\right)+\eta\left(x_{3}(t), y_{3}(t)\right)
$$

or equivalently,

$$
\begin{aligned}
& x(\xi, \eta, t)=(1-\xi-\eta) x_{1}(t)+\xi x_{2}(t)+\eta x_{3}(t), \\
& y(\xi, \eta, t)=(1-\xi-\eta) y_{1}(t)+\xi y_{2}(t)+\eta y_{3}(t) .
\end{aligned}
$$

Here we define $\mathbf{r}_{\mathbf{1}}=\left(x_{1}, y_{1}\right), \mathbf{r}_{\mathbf{2}}=\left(x_{2}, y_{2}\right)$ and $\mathbf{r}_{3}=\left(x_{3}, y_{3}\right)$. Substituting $\alpha_{i}, \beta_{i}, \tau_{i}$, (3.17) and (3.18) into (3.13)(3.15) results in 


$$
\phi_{1}(\xi, \eta)=1-\xi-\eta, \quad \phi_{2}(\xi, \eta)=\xi, \quad \phi_{3}(\xi, \eta)=\eta .
$$

The set $\left\{\phi_{1}, \phi_{2}, \phi_{3}\right\}$ is called a local (or nodal) basis for the set of linear functions. Therefore over each element $(e), \mathbf{U}$ reduces to

$$
\mathbf{U}^{(e)}(x(\xi, \eta, t), y(\xi, \eta, t), t)=\mathbf{U}^{(e)}(\xi, \eta, t)=\sum_{j=1}^{3} \mathbf{U}_{j}(t) \phi_{j}(\xi, \eta) .
$$

Under (3.17) and (3.18) the global linear basis functions (3.13)-(3.15) have been transformed from the global $(x, y)$ coordinates over each triangle to local basis linear functions $(3.19)$ in local $(\xi, \eta)$ coordinates. The Jacobian $\mathbf{J}$, of this transformation is constant over each element. Straightforward calculation gives

$$
\begin{aligned}
\mathbf{U}_{x}^{(e)} & =\frac{1}{|\mathbf{J}|}\left(\left(y_{3}(t)-y_{1}(t)\right)\left(\mathbf{U}_{2}^{(e)}-\mathbf{U}_{1}^{(e)}\right)-\left(y_{2}(t)-y_{1}(t)\right)\left(\mathbf{U}_{3}^{(e)}-\mathbf{U}_{1}^{(e)}\right)\right) \\
& =\frac{1}{|\mathbf{J}|}\left(\left(y_{2}(t)-y_{3}(t)\right) \mathbf{U}_{1}^{(e)}+\left(y_{3}(t)-y_{1}(t)\right) \mathbf{U}_{2}^{(e)}+\left(y_{1}(t)-y_{2}(t)\right) \mathbf{U}_{3}^{(e)}\right)
\end{aligned}
$$

and similarly

$$
\mathbf{U}_{y}^{(e)}=\frac{1}{|\mathbf{J}|}\left(\left(x_{3}(t)-x_{2}(t)\right) \mathbf{U}_{1}^{(e)}+\left(x_{1}(t)-x_{3}(t)\right) \mathbf{U}_{2}^{(e)}+\left(x_{2}(t)-x_{1}(t)\right) \mathbf{U}_{3}^{(e)}\right) .
$$

Although we have illustrated the finite element method with piecewise linear basis functions, quadratic or higher order forms that are more accurate could be used, though throughout we use linear basis functions as these are sufficient for our studies.

\subsection{The Galerkin method}

A function $\mathbf{u}(\mathbf{x})\left(\mathbf{x} \in \mathbb{R}^{2}\right)$ which is a solution to (2.1) that is $C^{2}$ in $\Omega(t)$, and is $C^{0}$ or $C^{1}$ on $\partial \Omega(t)$ (depending on whether the boundary conditions are Dirichlet or Neumann/Robin type) is called a classical solution. Multiplying (2.1) by $w \in H^{1}(\Omega(t))$ we have the following problem.

Find $\mathbf{u} \in H^{1}(\Omega(t))$ such that $\left(\mathbf{u}_{t}, w\right)=\left(\gamma \mathbf{f}+\mathbf{P}_{3}, w\right)+\mathbf{D}\left(\nabla^{2} \mathbf{u}, w\right)$ for all $w \in H^{1}(\Omega(t))$, where

$$
(\mathbf{u}, w)=\left(\iint_{\Omega(t)} u w \mathrm{~d} \mathbf{x}, \iint_{\Omega(t)} v w \mathrm{~d} \mathbf{x}\right)^{\mathrm{T}}
$$

is the $L_{2}$-inner product. Here we defined the Hilbert spaces $[1,11,21]$ by

$$
\begin{aligned}
& L_{2}(\Omega(t))=\left\{w, w \text { is defined on } \Omega(t) \text { such that } \int_{\Omega(t)}|w|^{2} \mathrm{~d} \mathbf{x}<\infty\right\}, \\
& H^{1}(\Omega(t))=\left\{w \in L_{2}(\Omega(t)) \frac{\partial w}{\partial \mathbf{x}_{i}} \in L_{2}(\Omega(t)), i=0, \ldots, N+1\right\} .
\end{aligned}
$$

Applying Green's Theorem and assuming that homogeneous Neumann boundary conditions are used, the weak form of the PDE system leads to the problem

Find $\mathbf{u} \in V^{h}$ such that $\left(\mathbf{u}_{t}, w\right)=\left(\gamma \mathbf{f}+\mathbf{P}_{\mathbf{3}}, w\right)+\mathbf{D} a(\mathbf{u}, w)$ for all $w \in V^{h} \subset H^{1}(\Omega(t))$, 
where $a(\mathbf{u}, w)=-\int_{\Omega(t)} \nabla \mathbf{u} . \nabla w \mathrm{~d} \mathbf{x}$. We substitute $\mathbf{u}$ with the finite element approximation $\mathbf{U}$. The Galerkin form then satisfies

$$
\left(\mathbf{U}_{t}, W\right)=\left(\gamma \mathbf{f}+\mathbf{P}_{\mathbf{3}}, W\right)+\mathbf{D} a(\mathbf{U}, W) \text { for all } W \in V^{h} \subset H^{1}(\Omega(t)) .
$$

Since the $W_{i}$ are coefficients of the test function, the Galerkin form also satisfies

$$
\begin{aligned}
& \left(\mathbf{U}_{t}, \varphi_{i}\right)=\left(\gamma \mathbf{f}+\mathbf{P}_{3}, \varphi_{i}\right)+\mathbf{D} a\left(\mathbf{U}, \varphi_{i}\right) \\
& \frac{\mathrm{d} \mathbf{U}_{j}}{\mathrm{~d} t}\left(\varphi_{j}, \varphi_{i}\right)-\left(\dot{x}_{j} \mathbf{U}_{x}+\dot{y}_{j} \mathbf{U}_{y}, \varphi_{i}\right)=\left(\gamma \mathbf{f}+\mathbf{P}_{3}, \varphi_{i}\right)+\mathbf{D} \mathbf{U}_{j} a\left(\varphi_{j}, \varphi_{i}\right)
\end{aligned}
$$

for all $i, j=1, \ldots, N+1$. $\mathbf{U}_{x}$ and $\mathbf{U}_{y}$ are defined in relations (3.21) and (3.22), respectively. $\dot{x}_{j}$ and $\dot{y}_{j}$ are prescribed. Over each element, we assemble matrices $m_{i, j} ; u x_{i, j} ; u y_{i, j} ; f_{i, j}, p_{3} i, j$ and $k_{i, j}, i, j=1,2,3$ called element matrices. All these element matrices are then assembled to give global matrices, leaving us with a time-dependent ordinary differential equation problem of the form

$$
M \dot{\mathbf{U}}+\mathbf{D} K \mathbf{U}=(\gamma F+P)(\mathbf{U})+(U X+U Y) \mathbf{U}
$$

where $M$ is the mass matrix, $F$ and $P$ result from the nonlinear terms (these depend on $\mathbf{U}$ ), $K$ is the global stiffness matrix and $U X$ and $U Y$ are the global matrices resulting from grid movement. If the domain is fixed in time, $U X$ and $U Y$ are zero matrices. Solving (3.28) gives us an approximation to the classical solution $\mathbf{u}$.

\subsection{Time-stepping}

The discretisation of the time derivative and the linearisation of nonlinear terms are carried out simultaneously. The nonlinear terms can be written in matrix-vector-product form where the matrix depends on either $U$ or both $U$ and $V$ (for specific details, see [24]). This form makes it possible to linearise the nonlinear terms by use of the Picard iteration method [38]. For example, the term $U^{2}$, can be written as a product of $U^{m} U^{m+1}$ where $U^{m}$ is the known solution calculated from the previous time $m \Delta t$. On a continuously moving grid this will be equivalent to calculating the successive solution $U^{m+1}$, where the previous solution $U^{m}$ is evaluated on the new grid. The Picard iteration method is combined with the implicit backward Euler finite difference scheme to yield a robust and stable scheme. To solve the set of linear algebraic equations obtained, a preconditioned generalised minimum residual method is used. Preconditioners such as ILU(0), ILUT, ILUTP, ILUK and MILU(0) can be used with the method [39,40]. In all our simulations we employ ILU(0). The criterion for convergence to the inhomogeneous steady state and hence a stopping criterion is

$$
\sqrt{\frac{\sum\left|\mathbf{U}^{m+1}-\mathbf{U}^{m}\right|^{2}}{\sum\left|\mathbf{U}^{m+1}\right|^{2}}} \leqslant \epsilon
$$

with $\epsilon$ typically of the order $10^{-6}-10^{-9}$.

\section{Transient solutions on regular and irregular growing domains}

We investigate the role of domain growth on pattern selection and transition on regular and irregular growing domains. The assumption is that the evolution of the solutions of the model equations takes place on the same timescale as domain growth. In Section 3, we pointed out that domain growth is prescribed. 
Biologically we assume that the nodal movement can be derived from experimental data. However, for illustrative purposes, we prescribe specific growth functions that continuously deform the domain.

One of the simplest ways to do this is to assume that the boundary deforms uniformly. Although there is no biological justification for this type of growth, Crampin et al. [15] studied and presented numerical results on uniform and non-uniform domain growth in one dimension. Hence we consider that the nodal movement can be written as a product of two functions, one that depends on the initial grid only and another that depends on time only. We can therefore define $x(t)=X r(t)$ and $y(t)=\operatorname{Yr}(t)$, to represent the boundary grid movement where $X$ and $Y$ are the initial boundary grid coordinates of $x(0)$ and $y(0)$, respectively, at time $t=0$. The velocity of grid movement is given by $\dot{x}(t)=X \dot{r}(t)$ and $\dot{y}(t)=Y \dot{r}(t)$. The above is valid if the function $r(t)$ satisfies the property $r(0)=1$ at time $t=0$. For illustrative purposes we will choose an exponential function of the form $r(t)=\mathrm{e}^{\sigma t}, t \geqslant 0$. One of the assumptions on domain growth is that it is slow and for this we require that $\sigma$ be a small parameter. This form of domain growth has been used previously in the study of robust pattern formation under domain growth [14,36,37]. In their studies $\sigma$ is assumed to be of the order $10^{-2}-10^{-5}$. For the sake of comparison with their work where possible, we assume that the parameter is of the same magnitude. Observe that the boundary continuously deforms. The new positions of the internal grid coordinates need to be calculated: here, we apply a spring analogy [10] to calculate the internal mesh movement. The spring analogy is a common technique for deforming a mesh, and consists of replacing the mesh by fictitious springs. There are two types of springs, the vertex and the segment spring analogies. The vertex spring is used mainly to refine a particular mesh, while the segment spring is appropriate for deforming and moving a given mesh. This technique preserves the number of grid coordinates and the mesh connectivity which enables smooth evolution. The initial mesh and the boundary grid points are generated by a Delaunay mesh generator [31]. All the numerical simulations are carried out on a typical mesh of approximately 4000 elements with approximately 2000 nodes.

In all our simulations the pattern transition on growing domains is independent of numerical parameters such as mesh size and time-step. By refining the mesh and time-step sizes, numerical solutions remain qualitatively similar with only small quantitative changes occurring. Numerical simulations have been carried out with different time-steps and different mesh sizes and the transition process was observed to be qualitatively unchanged. Numerical convergence for our method can be shown in terms of mesh grid refinement and at least first order rate of convergence with respect to the number of mesh points has been observed (see [24]).

In this paper, we plot the contour profiles of the inhomogeneous steady-state solutions only. This is mainly for biological interpretation. In the biological context colour patterns are linked to cell differentiation. Assuming that coloration is determined by a constant threshold value in $u$ (or $v$ ) concentration, $u_{s}$ (or $v_{s}$ ) say, such that cells in the region where $u \geqslant u_{s}$ are black, while cells which experience a concentration $u<u_{s}$ are coloured white, these give rise to the shading illustrated in all the figures. The actual solution surfaces $(u$ or $v)$ are as depicted in Fig. 1.

\subsection{Transient solutions on regular domains: Schnakenberg kinetics}

\subsubsection{Stripe-to-stripe patterns}

For illustrative purposes, we solve the Schnakenberg model equations with homogeneous Neumann boundary conditions applied to both $u$ and $v$. Let us first consider square and rectangular domains. The simplest form of growth is when it occurs along one axis of the domain. This can be viewed as an extension of one-dimensional domain growth where values of $x$ or of $y$ are fixed as constants. A typical example of this growth is shown in Figs. 2(a) and (b). We let the initial unit square grow exponentially along the $x$-axis until it reaches the final domain, taken here to be $[0,6] \times[0,1]$. The numerically computed transient solutions of the model equations on a growing unit square in this example consist of an elongating stripe. From linear stability theory (Appendix A), the parameter values of $\gamma$ and $d$ are selected such that the $(1,0)$ 

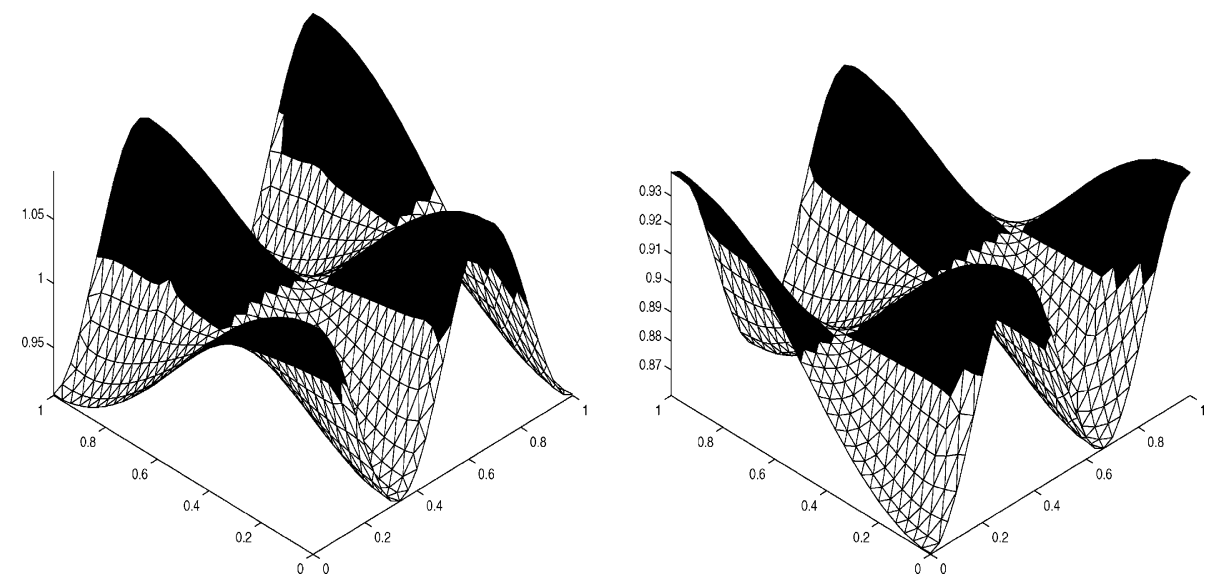

Fig. 1. The solution surfaces of $u$ and $v$ illustrating the type of shading used.

mode is isolated on a unit square. Linear stability theory predicts that $(0,1)$ is always an excitable mode on the domain $[0, L] \times[0,1]$ for any length $L$. The corresponding eigenfunction of the Laplacian with homogeneous Neumann boundary conditions on this domain can be shown to be given by $\cos \pi y$. Hence the stripe pattern produced from the transient solutions is consistent with predictions from linear stability theory.

If, however we prescribe initial conditions which are biased towards the pattern generated by the eigenfunction $\cos \pi x$ on a unit square, we obtain the numerical solution illustrated in Figs. 2(c)-(e). As the unit square continues to grow exponentially along the $x$-axis, the transient solution changes orientation. The domain is grown until it reaches the size $[0,8] \times[0,1]$. In both simulations, the numerical solution of the model equations under this form of domain growth with these parameter values produces stripe

(a)

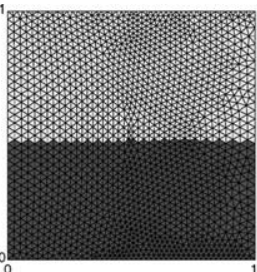

(c)

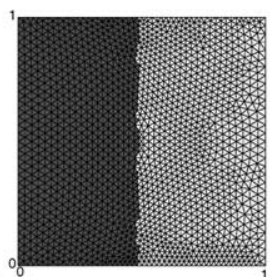

(b)

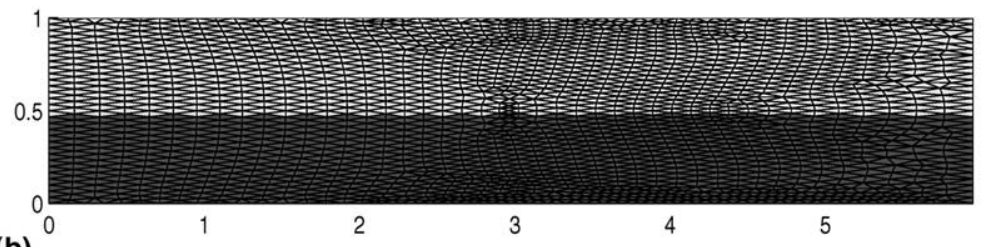

$\begin{array}{ll}\text { (d) } & \text { (e) }\end{array}$

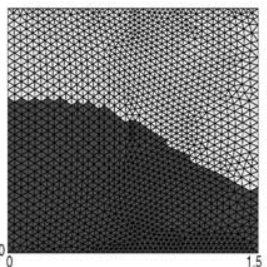

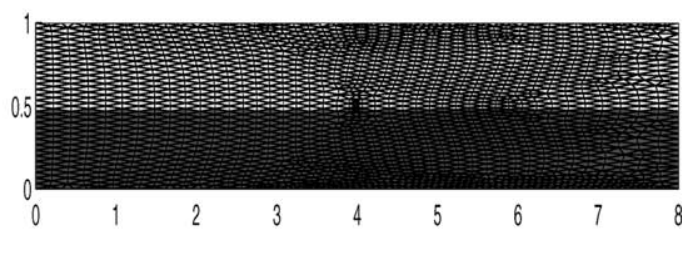

(e)

Fig. 2. The domain $[0,1] \times[0,1]$ is grown exponentially to reach the final domain $[0,6] \times[0,1]$. The Schnakenberg model is solved with parameter values $a=1.0, b=0.9, \gamma=29$ and $d=10$. (a,b) A stripe is generated and simply elongates as the domain grows along the $x$ axis. (c)-(e) The unit square domain is grown exponentially to reach the final $[0,8] \times[0,1]$ domain. Initial conditions are chosen such that a stripe parallel to the $y$-axis is produced. The orientation of the pattern changes completely as the domain grows, a stripe emerges perpendicular to the $y$-axis, recapturing the orientation in (b). 


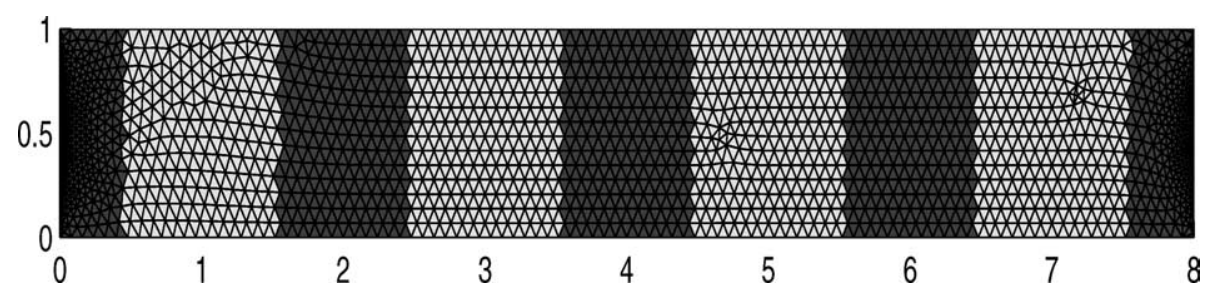

Fig. 3. Numerically computed spatially inhomogeneous steady-state solution on a fixed domain $[0,8] \times[0,1]$ with random initial conditions. This type of solution is not observed on a growing unit square as shown in Figs. 2(a)-(e).

patterns independent of initial conditions. This indicates that pattern selection under domain growth can be insensitive to initial conditions when these are small amplitude perturbations about the uniform steady state.

In order to highlight the crucial role of growth in pattern selection we illustrate in Fig. 3 the result of solving the model equations on a fixed domain with homogeneous Neumann boundary conditions and a random initial condition but with exactly the same parameter values as in Fig. 2. Instead of horizontal stripes, vertical stripes are obtained. This solution is not computed nor observed under the domain growth of Fig. 2, and quite possibly under no form of dynamic domain elongation. Thus it is apparent that domain growth enhances the robustness of pattern selection.

The concept of robustness of pattern selection and transition is still to be fully understood and conceptualised. In this simple case, we can show by use of linear stability analysis how domain growth enhances the robustness of pattern formation. From linear stability theory, the parameter values are selected such that the $(0,1)$ (or equally $(1,0)$ ) mode is isolated on a unit square. That is the uniform steady state can only go unstable to this unique (up to $\pi / 2$ rotational symmetry of the domain) admissible mode which has a positive growth rate. Once the parameter values are selected, these are fixed as the unit square deforms. If it is deformed continuously along the $x$-axis, the $(0,1)$ mode is always an admissible mode but it is not the only admissible mode. Once the $(0,1)$ mode has been selected and nonlinearly evolves, other growing modes appear not to be relevant. However if the domain is fixed, as shown in Fig. 3, the growth rate of the $(0,1)$ mode is less than the growth rate of some other acceptable mode and it is therefore less likely to be observed or computed. The spatially inhomogeneous solution obtained will crucially depend on the initial conditions chosen. We remark that analytical linear stability theory becomes nontrivial and complicated for mixed modes. Moreover, it cannot address the problem of nonlinear pattern selection, for which no standard theory exists.

\subsubsection{Stripe-to-spot patterns}

Let us consider now a different form of growth from the essentially one-dimensional form described previously. Suppose we let the unit square grow along the diagonal giving rise to successively larger square domains.

For illustrative purposes, we choose parameter values determined to isolate the $(1,0)$ mode on a unit square. The transient solution of the model equations with these parameter values on a growing unit square are shown as the sequence of frames in Fig. 4. As the domain grows a stripe pattern transforms into a spot pattern. This evolution process occurs through peak insertion, whereby a stripe evolves into a spot pattern. Further domain growth shows peak splitting from a single spot to four spots. This result has been observed before [14].

Hence we have observed in one simulation the insertion and splitting of peaks. As the evolution of four spots continues they merge to form oblique stripes due to peak insertion. When the growing domain reaches $[0,3.5] \times[0,3.5]$, a circular pattern is observed. On further growth, the domain passes through 

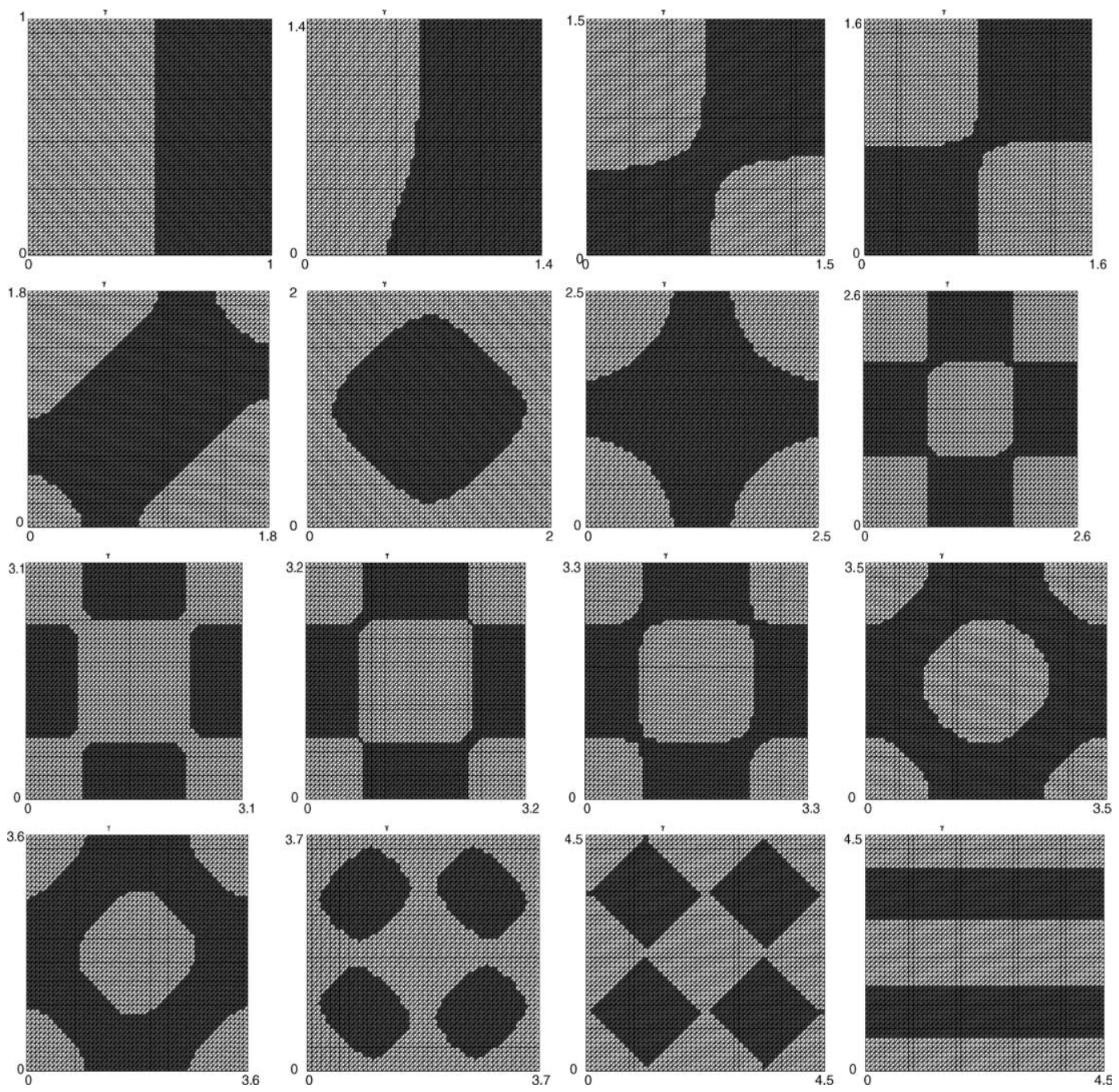

Fig. 4. Transient patterns generated by the Schnakenberg model as the unit square is grown along the diagonal line $x=y$ at constant speed in the positive direction. The parameter values in the numerical computations are $a=0.1, b=0.9, d=10$ and $\gamma=10$. Stripes evolve to spots which in turn evolve into circular patterns as the domain grows. Further growth transforms these back into spots. However, when domain growth is stopped, the spatially inhomogeneous steady-state solution computed to which the system converges is that of stripes.

another transition point. Although the circular pattern is still more pronounced on the domain $[0,3.6] \times[0,3.6]$, we observe peak insertion as the domain grows. This gives rise to the formation of spots as the circular pattern breaks down. Spots are illustrated more clearly on the domains from $[0,3.7] \times[0,3.7]$ up to the domain $[0,4.5] \times[0,4.5]$. When the domain reaches the final domain size, taken here to be $[0,4.5] \times[0,4.5]$, the simulation converges to a spatially inhomogeneous steady state on this fixed domain. Surprisingly, stripes are produced in favour of spots. It appears therefore that growth stabilises spots over stripes. It certainly indicates that dynamic growth significantly affects patterning. However more detailed and extensive numerical experiments are required to confirm this. Different random initial conditions have been prescribed as small perturbations about the uniform steady state and we have observed qualitatively 
similar sequences, hence we conclude that pattern transition under domain growth is insensitive locally to initial data.

\subsubsection{Spot-to-stripe-to-spot patterns}

Fig. 5 illustrates a pattern sequence in which spots and stripes are produced in an alternating fashion. Parameters are chosen such that linear theory predicts evolution to the $(2,2)$ mode on the unit square. The corresponding eigenfunction is given by $\cos (2 \pi x) \cos (2 \pi y)$ with eigenvalue $k_{2,2}=8 \pi^{2}$. The pattern shown in Fig. 5(a) is obtained from the numerical solution of the model equations on a unit square and is consistent with this eigenfunction.

When the unit square is grown exponentially along the diagonal $y=x$ to reach the domain $[0,1.6] \times[0,1.6]$ it passes through a region where spots become inadmissible patterns. Instead of spots, stripes are formed (Fig. 5(b)). This process is independent of the initial conditions. We have carried out numerical tests with different initial conditions and with more refined grids and obtained the same results. On further growth, up to $[0,1.8] \times[0,1.8]$, the reverse takes place, stripes becoming inadmissible while spots are generated, as shown in Fig. 5(c). With further growth, this process is repeated (Figs. 5(d)-(f)). The pattern obtained in Fig. 5(e) compares qualitatively with the predictions from linear theory that the eigenfunction $\cos [(6 \pi / 2.4) y]$ of the Laplacian with zero-flux boundary conditions on this domain should grow. If domain growth stops, at say $[0,2.8] \times[0,2.8]$, and the simulations allowed to converge, the pattern produced is a mixture of spots and stripes.

So far we have investigated the role of domain growth by growing a unit square domain exponentially either along one of the axes to produce rectangular domains or along one of the diagonals producing square domains. We have extended this study to a number of growing domains including a right-angled isosceles triangle, an equilateral triangle and a hexagon [24]. We present results only for the growing hexagon. We comment that solutions on a growing equilateral triangle display one of two properties; either they are symmetrical with respect to a $120^{\circ}$ rotation about the centre or they are symmetrical about an altitude.

(a) ${ }^{0}$

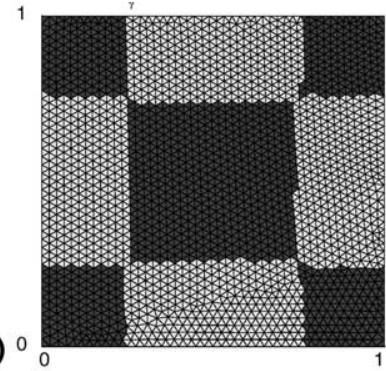

(d)

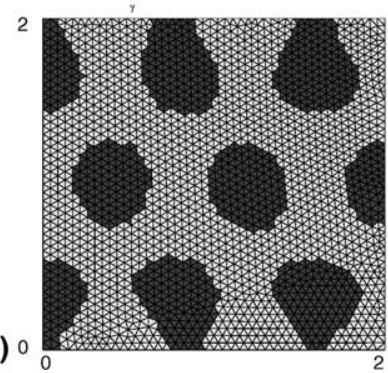

(b) 0

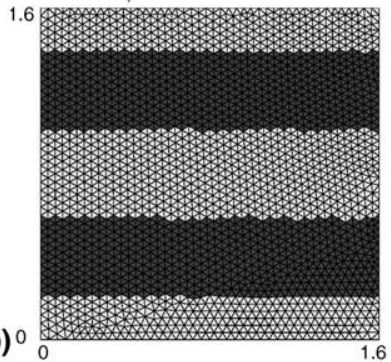

(c) ${ }^{0}$

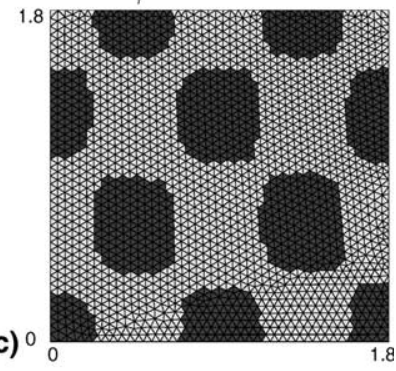

(e) 0

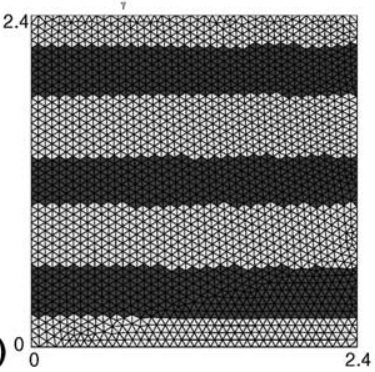

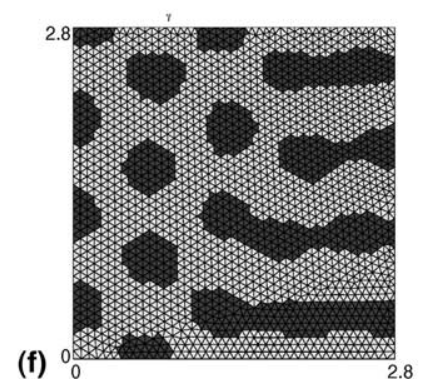

Fig. 5. Transient solutions of the Schnakenberg model on the unit square growing along the diagonal $y=x$. The parameter values are chosen such that the $(2,2)$ mode is isolated on a unit square. These are given as $a=0.1, b=0.9, d=8.6676$ and $\gamma=230.82$. The observed transient patterns are spots, stripes and spots alternating repeatedly as the domain grows. 


\subsubsection{Transient patterns on a hexagon}

We investigate the role of domain growth on a hexagon of unit side given by the vertices $(1,0) ;\left(\frac{1}{2}, \frac{\sqrt{3}}{2}\right)$; $\left(-\frac{1}{2}, \frac{\sqrt{3}}{2}\right) ;(-1,0) ;\left(-\frac{1}{2},-\frac{\sqrt{3}}{2}\right)$ and $\left(\frac{1}{2},-\frac{\sqrt{3}}{2}\right)$. The hexagon is allowed to grow exponentially from its centre to three times its original size. Fig. 6 shows the corresponding numerical solutions of the model system with homogeneous Neumann boundary conditions. The parameter values are unchanged from those used on the equilateral triangle.

(a)

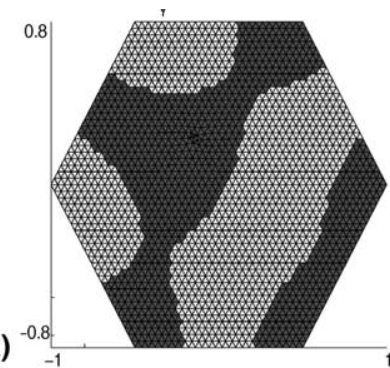

(d)

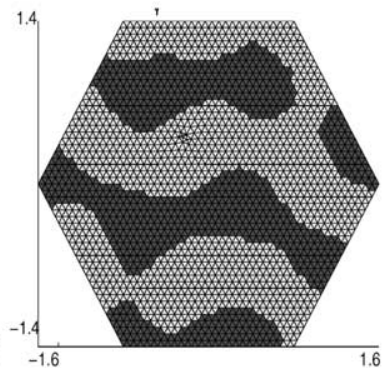

(g)

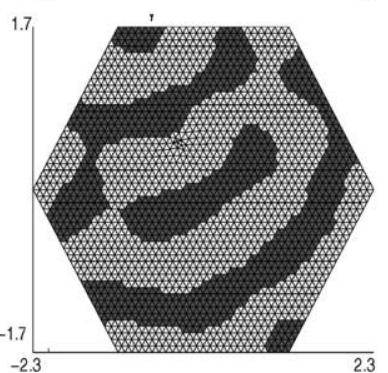

(h)

(e)

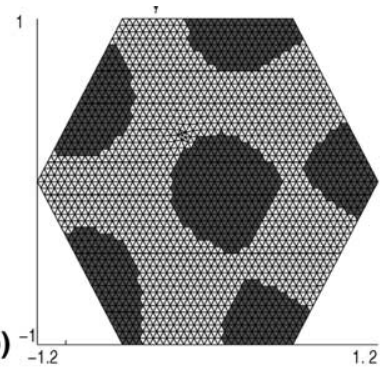

-1. S
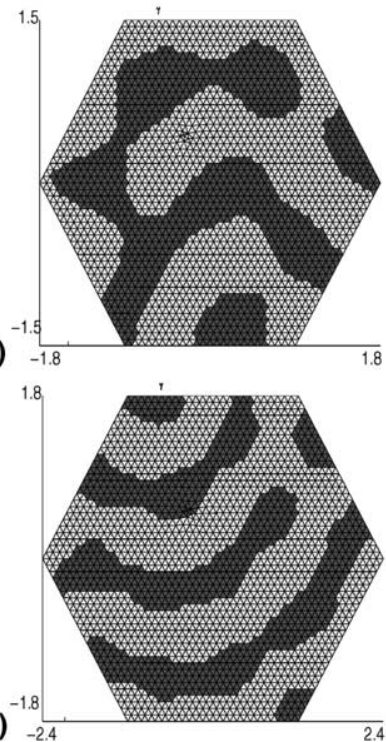

(f)

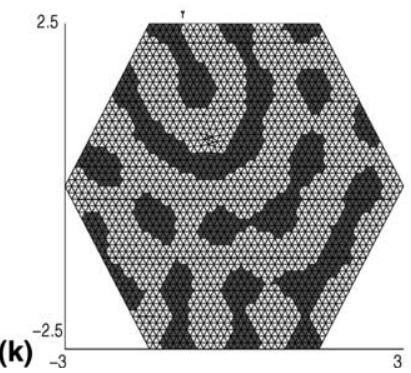

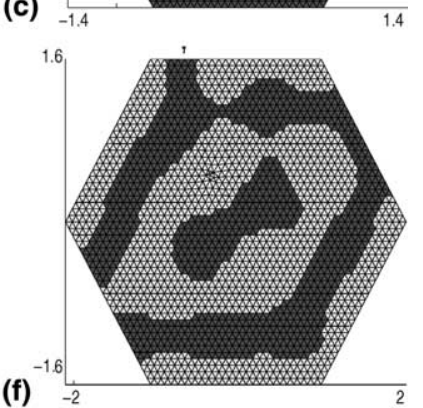
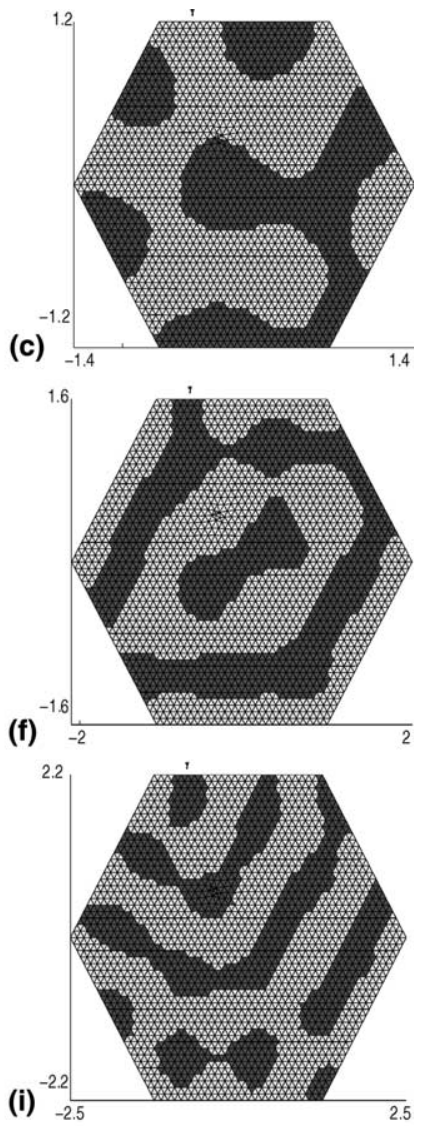

(i)

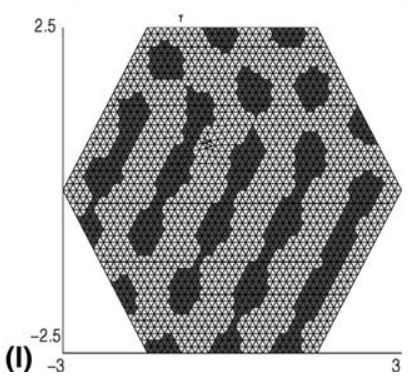

Fig. 6. Typical numerically computed patterns on a growing hexagon for the Schnakenberg model with parameter values $a=0.1$, $b=0.9, d=10$ and $\gamma=114$. Stripe, spot and circular patterns are exhibited. Circular patterns become pronounced as the hexagon grows in size. As the domain reaches its maximum size, the system evolves to an inhomogeneous steady-state solution consisting of stripes and spots. 


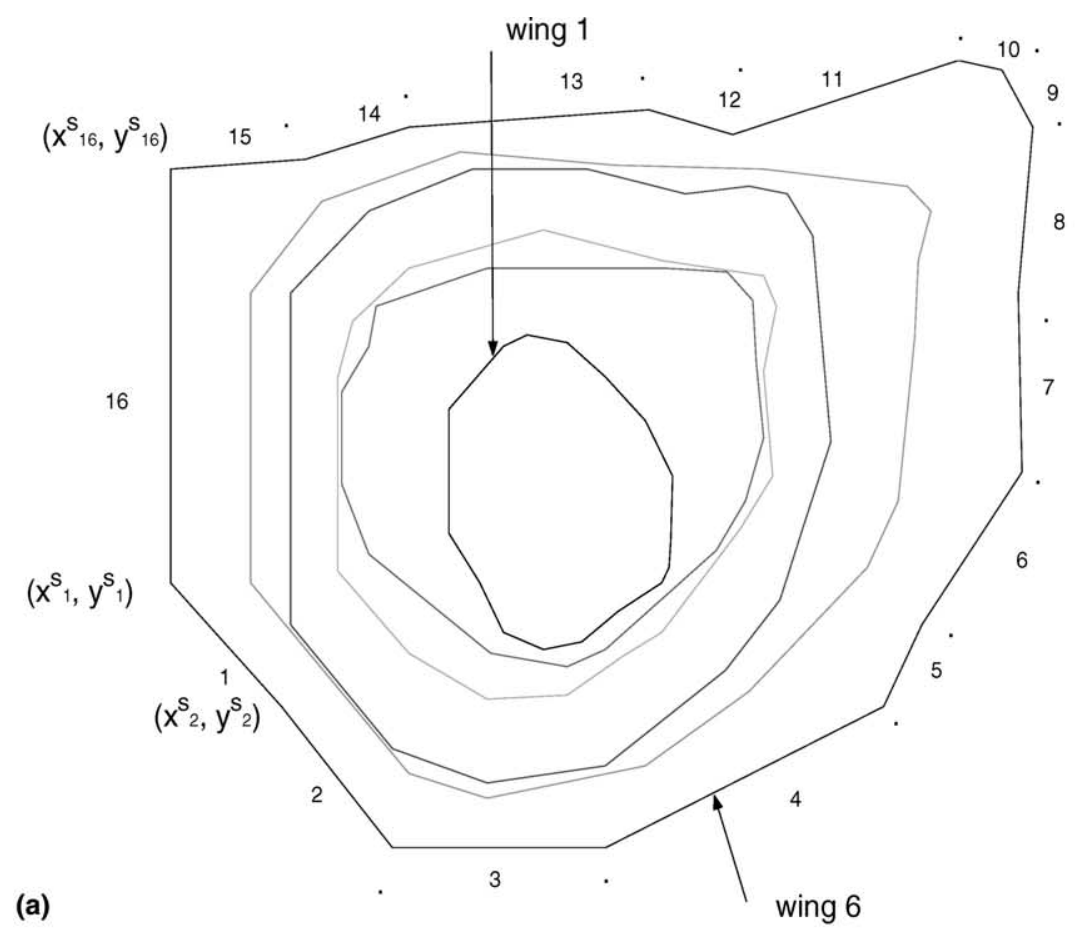


Initially we observe stripes (Fig. 6(a)) evolving to spots as the domain grows as shown in Fig. 6(b). These spots further evolve to stripes (Figs. 6(c) and (d)). A mixed mode solution then gives rise to circular patterns as illustrated in the sequence shown in Figs. 6(e)-(k). The circular patterns continue to evolve as the domain deforms continuously until it reaches its final size. On this fixed domain the spatially inhomogeneous steady-state pattern obtained is that of stripes and spots combined (Fig. 6(1)). The sequence illustrated is independent of the initial conditions.

Although this sequence exhibits several features in common with our previous simulations above, a new pattern is observed, namely circular patterns. The appearance of such patterns seems to be due to the nature of the geometry. The circular patterns appear as transient solutions when stripes and spots occur at almost the same time but neither solution dominates due to domain growth. Such patterns have been observed in zebrafish [37] and Pomacanthus [22]. This phenomenon could explain the emergence of circular patterns of pigmentation on fish which takes place as the fish grows in size. A combination of spots and stripes are observed when the zebrafish attains its maximum size, which compares qualitatively with the inhomogeneous steady-state pattern computed on the final fixed domain. When the domain reaches its final size the numerical solution converges to a mixed pattern of stripes and spots (Fig. 6(1)). The evolution of the transient solutions on the growing domain is insensitive to the initial conditions. Therefore under domain growth the model is robust as the patterns generated are independent of the initial conditions, when these are small amplitude perturbations about the uniform steady state.

\subsection{Transient solutions on irregular domains: Gierer-Meinhardt kinetics}

The aim of this section is to illustrate the generality of our numerical technique in dealing with arbitrary simply connected domains. This work was motivated by the study of growth colour patterns in the butterfly wing Papilio dardanus. The biological background to the mathematical problem is well documented in the papers by Sekimura et al. [43-45] and in the book by Nijhout [34]. Our aim here is not to compute patterns typically observed in biological experiments but to illustrate the generality of our numerical method.

The problem here is, given a discrete sequence of the wing size and shape of the butterfly $P$. dardanus can we predict and calculate the evolving patterning process? Typically the discrete sequence is given by the scans at the following time intervals, 12, 36, 48, 60, 72 and $96 \mathrm{~h}$ (Fig. 7 and Table 1). Interpolation between

Table 1

The boundary coordinates of the 16-sided polygonal domain of the six wings in Fig. $7^{\text {a }}$

\begin{tabular}{lllllll}
\hline Time $(\mathrm{s})$ & $0.432 \times 10^{5}$ & $1.296 \times 10^{5}$ & $1.728 \times 10^{5}$ & $2.16 \times 10^{5}$ & $2.59 \times 10^{5}$ & $3.456 \times 10^{5}$ \\
\hline$\left(x^{s_{1}}, y^{s_{1}}\right)$ & $(-0.43,-0.39)$ & $(-1.02,-0.13)$ & $(-1.04,-0.59)$ & $(-1.3,-0.87)$ & $(-1.52,-0.65)$ & $(-1.96,-0.65)$ \\
$\left(x^{s_{2}}, y^{s_{2}}\right)$ & $(-0.26,-0.65)$ & $(-0.87,-0.5)$ & $(-0.65,-1.02)$ & $(-0.74,-1.52)$ & $(-0.65,-1.65)$ & $(-1.35,-1.3)$ \\
$\left(x^{s_{3}}, y^{s_{3}}\right)$ & $(-0.13,-0.91)$ & $(-0.2,-1.02)$ & $(-0.22,-1.26)$ & $(-0.22,-1.7)$ & $(-0.22,-1.78)$ & $(-0.74,-2.04)$ \\
$\left(x^{s_{4}}, y^{s_{4}}\right)$ & $(0.09,-1.0)$ & $(0.22,-1.09)$ & $(0.22,-1.24)$ & $(0.43,-1.61)$ & $(0.65,-1.61)$ & $(0.43,-2.04)$ \\
$\left(x^{s_{5}}, y^{s_{5}}\right)$ & $(0.3,-0.96)$ & $(0.43,-1.0)$ & $(0.52,-1.04)$ & $(1.09,-1.11)$ & $(1.22,-1.22)$ & $(1.96,-1.3)$ \\
$\left(x^{s_{6}}, y^{s_{6}}\right)$ & $(0.5,-0.8)$ & $(1.04,-0.48)$ & $(0.74,-0.91)$ & $(1.39,-0.74)$ & $(1.87,-0.57)$ & $(2.17,-0.87)$ \\
$\left(x^{s_{7}}, y^{s_{7}}\right)$ & $(0.74,-0.65)$ & $(1.2,-0.22)$ & $(1.17,-0.37)$ & $(1.52,-0.35)$ & $(2.04,-0.22)$ & $(2.72,-0.07)$ \\
$\left(x^{s_{8}}, y^{s_{8}}\right)$ & $(0.78,-0.57)$ & $(1.3,0.11)$ & $(1.35,-0.09)$ & $(1.67,0.09)$ & $(2.13,0.63)$ & $(2.7,0.87)$ \\
$\left(x^{s_{9}}, y^{s_{9}}\right)$ & $(0.79,-0.43)$ & $(1.26,0.5)$ & $(1.3,0.46)$ & $(1.57,1.17)$ & $(2.15,1.04)$ & $(2.78,1.74)$ \\
$\left(x^{s_{10}}, y^{s_{10}}\right)$ & $(0.8,-0.09)$ & $(1.24,0.83)$ & $(1.37,0.8)$ & $(1.43,1.39)$ & $(2.22,1.3)$ & $(2.61,2.04)$ \\
$\left(x^{s_{11}}, y^{s_{11}}\right)$ & $(0.65,0.2)$ & $(1.1,0.98)$ & $(1.3,0.96)$ & $(1.22,1.43)$ & $(2.09,1.43)$ & $(2.37,2.09)$ \\
$\left(x^{s_{12}}, y^{s_{12}}\right)$ & $(0.43,0.43)$ & $(0.76,1.0)$ & $(0.74,1.04)$ & $(0.87,1.39)$ & $(1.30,1.52)$ & $(1.13,1.7)$ \\
$\left(x^{s_{13}}, y^{s_{13}}\right)$ & $(0.22,0.61)$ & $(-0.22,1.0)$ & $(0.09,1.2)$ & $(0.33,1.52)$ & $(0.4,1.54)$ & $(0.67,1.83)$ \\
$\left(x^{s_{14}}, y^{s_{14}}\right)$ & $(0.0,0.65)$ & $(-0.83,0.8)$ & $(-0.65,1.0)$ & $(-0.3,1.52)$ & $(-0.37,1.61)$ & $(-0.65,1.74)$ \\
$\left(x^{s_{15}}, y^{s_{15}}\right)$ & $(-0.13,0.59)$ & $(-0.87,0.59)$ & $(-0.96,0.72)$ & $(-0.87,1.3)$ & $(-1.13,1.35)$ & $(-1.22,1.57)$ \\
$\left(x^{s_{16}}, y^{s_{16}}\right)$ & $(-0.43,0.26)$ & $(-1.02,0.35)$ & $(-1.04,0.43)$ & $(-1.3,0.87)$ & $(-1.52,0.87)$ & $(-1.96,1.52)$ \\
\hline
\end{tabular}

${ }^{\mathrm{a}}$ Here, $s_{k}$ represents the successive locations of the $(x, y)$ end points on each segment of the polygon. 
the positions of the boundary nodes of the boundary shape is achieved with cubic splines thus defining a continuously deforming domain. Once the boundary position of the continuously deforming boundary is located, the spring analogy is applied at each time step to define the internal mesh and hence to define the motion on the internal nodes.

We solve the Gierer-Meinhardt reaction kinetics with homogeneous Neumann boundary conditions for both $u$ and $v$. We note that these are not realistic boundary conditions for the butterfly pattern [45]. Initial conditions are taken as random perturbations about a constant value. Figs. 8 and 9 show the results of such computations. As the domain grows continuously transient patterns evolve from spots to circular patterns. The scale changes in Figs. 8 and 9 are small compared to the rest of the previous figures. Therefore we plot the figures on the same scale $[-2,2.6] \times[-2,2]$ and this is not shown in the illustrated figures.

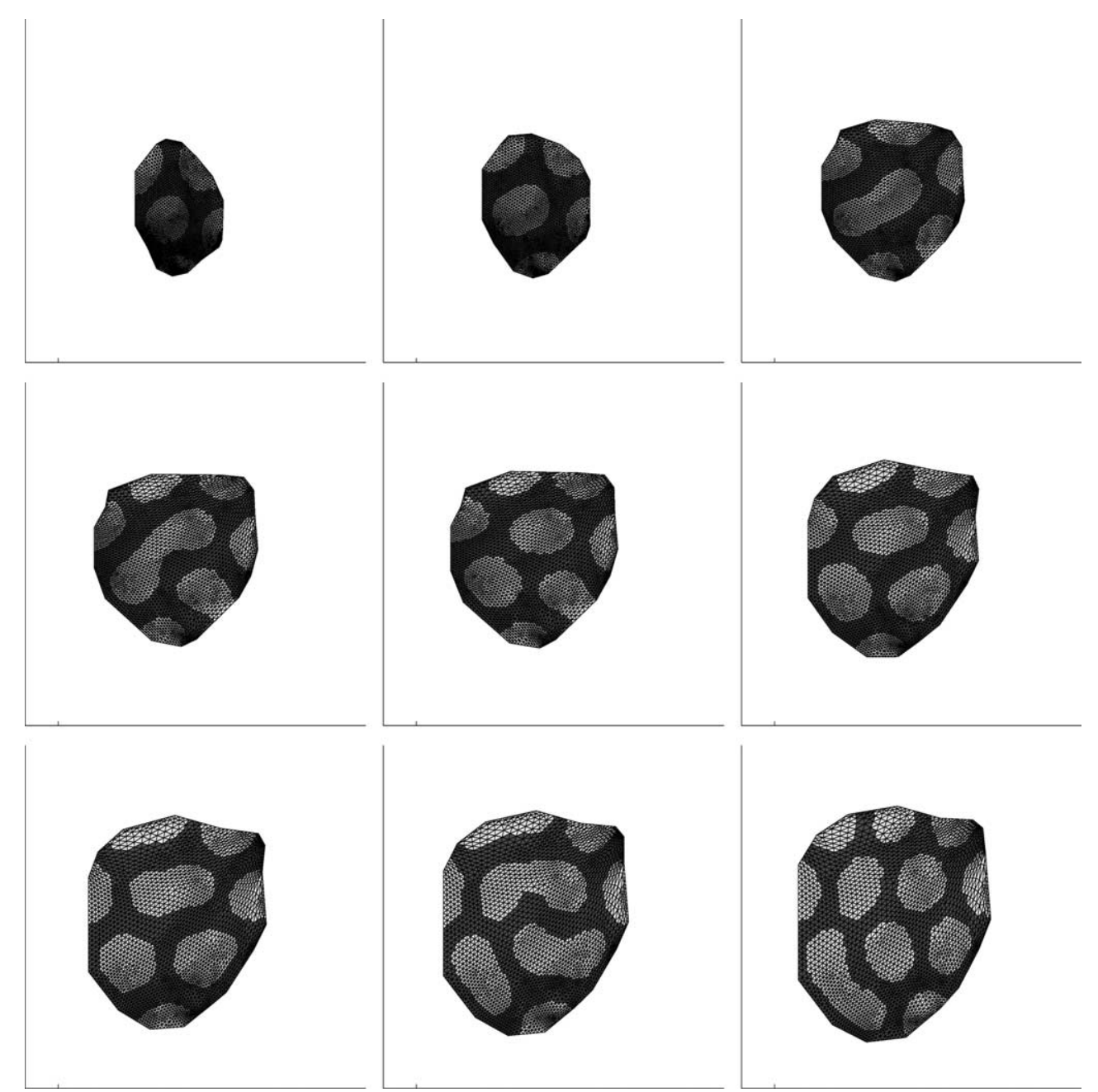

Fig. 8. Transient patterns of the solution $u$ corresponding to the Gierer-Meinhardt reaction model with parameter values $a=0.1$, $b=1.0, K=0.5, d=70.8473$ and $\gamma=619.45$. Spot patterns continue to evolve with domain growth. The numerical simulations are carried out on a typical mesh of 4730 elements with 2466 nodes. 

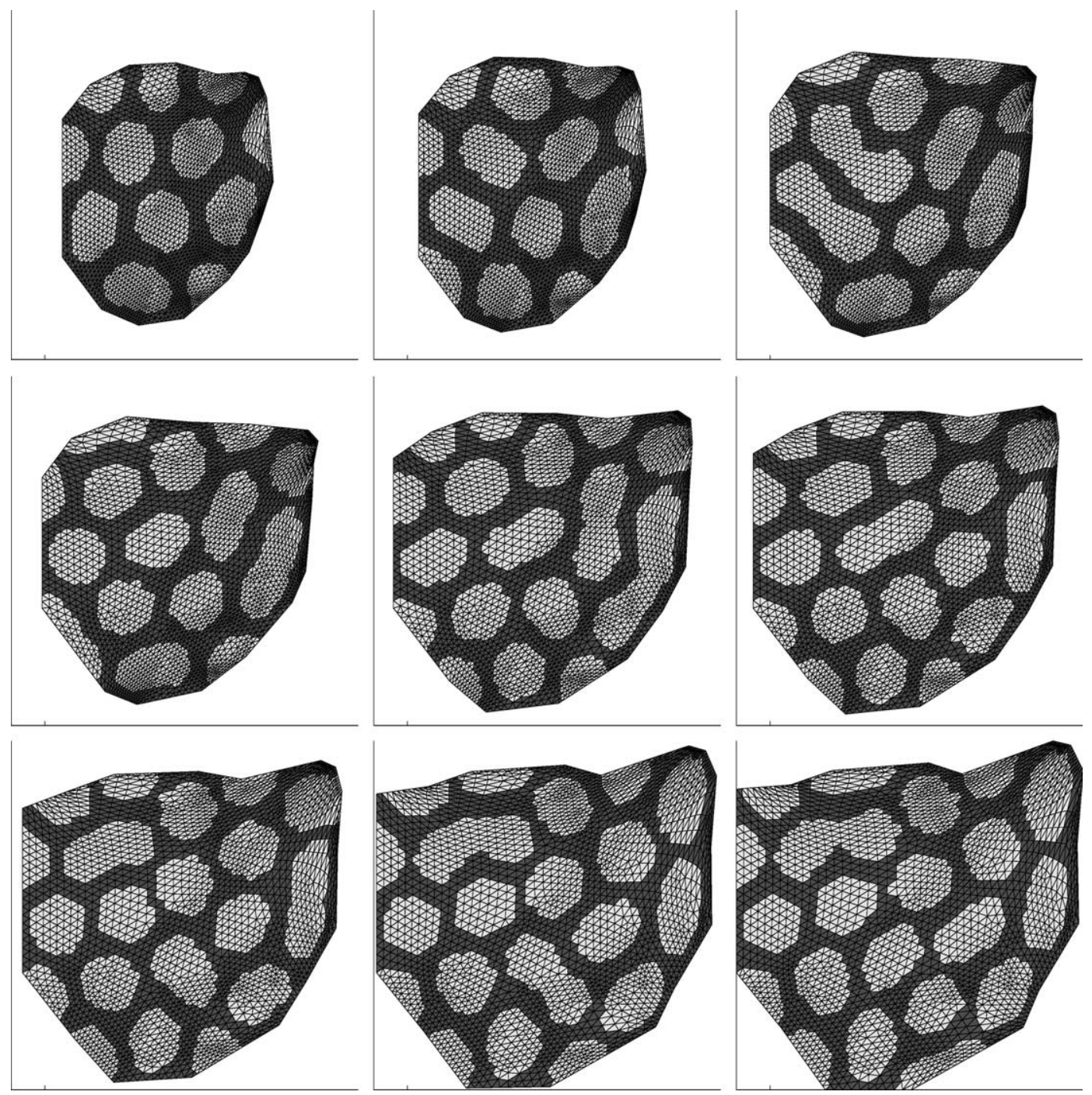

Fig. 9. Fig. 8 continued. As the domain continuously deforms, a combination of stripes and spots is exhibited on the same domain.

\section{Conclusion and discussion}

The novel application of the moving grid finite element method to reaction-diffusion systems on a continuously deforming domain has opened new avenues in the study of pattern formation and transition in Biology. In particular, we have developed a freely available software package that can be used not only to investigate pattern formation, but also to predict pattern transition. All results reported in this paper are produced with this package and it can be downloaded from: http://web.comlab.ox.ac.uk/oucl/work/andy.wathen/software.html.

This technique has been applied to the study of colour pattern formation of the butterfly wing $P$. dardanus $[25,26,45]$, and growth patterns in bivalve ligaments $[25,26,48]$. 
We have observed that stripes can evolve into stripes, spots into spots and sometimes stripes evolve into spots which in turn involve into circular patterns. The emergence of such exotic patterns is due to domain growth. Domain growth appears to enhance robustness in pattern selection and transition: lack of robustness has been one of the main criticisms of the Turing theory of pattern formation on fixed domains.

The generality of our numerical method allows us to carry out further investigative real studies into pattern transition in biological problems. Currently we are investigating growth patterns of the butterfly wing $P$. dardanus and of the zebrafish.

\section{Acknowledgements}

This work (AM) was supported by a grant from EPSRC Life Sciences Initiative (GR/R03914). PKM was partly supported by a Royal Society Leverhulme Trust Senior Fellowship. We thank Professor Toshio Sekimura (Chubu University, Japan) for bringing the problem of butterfly pigmentation patterning to our attention and for providing the data used in Fig. 7.

\section{Appendix A. Linear analysis}

Standard linear stability analysis shows that diffusion-driven instability of a uniform steady state of the systems (2.2)-(2.8) occurs if the following conditions hold (see, for example, the books by [16,33]:

$$
\begin{aligned}
& f_{u}+g_{v}<0, \\
& f_{u} g_{v}-f_{v} g_{u}>0, \\
& \mathrm{~d} f_{u}+g_{v}>0, \\
& \left(\mathrm{~d} f_{u}+g_{v}\right)^{2}-4 \mathrm{~d}\left(f_{u} g_{v}-f_{v} g_{u}\right)>0,
\end{aligned}
$$

where the partial derivatives are evaluated at the steady state. Inequalities (A.1)-(A.4) define a domain of parameter space, known as the Turing space, wherein the uniform steady state is unstable to random small perturbations.

Under these conditions, spatial disturbances with wavenumbers $k \in\left(k_{-}, k_{+}\right)$will initially grow, where

$$
k_{ \pm}^{2}=\gamma \frac{\left(\mathrm{d} f_{u}+g_{v}\right) \pm \sqrt{\left(\mathrm{d} f_{u}+g_{v}\right)^{2}-4 \mathrm{~d}\left(f_{u} g_{v}-f_{v} g_{u}\right)}}{2 d} .
$$

In the case we are interested in, namely, the unit square with zero flux boundary conditions, a further restriction on $k$ is that it must take discrete values $\pi \sqrt{m^{2}+n^{2}}$, corresponding to the spatial mode $\cos m \pi x \cos n \pi y$ denoted by $(m, n)$.

For diffusion-driven instability to occur we require that the Turing conditions (A.1)-(A.4) are satisfied and if we wish to isolate a certain mode, say $(m, n)$, then

$$
k_{\max }^{2} \leqslant k_{-}^{2}<k_{m, n}^{2}=\pi^{2}\left(m^{2}+n^{2}\right)<k_{+}^{2} \leqslant k_{\min }^{2},
$$

where

$$
k_{\max }^{2}=\max \left\{\forall k_{p, q}^{2}: k_{p, q}^{2}<k_{m, n}^{2} \text { for some } p, q\right\},
$$


Table 2

Values for $d$ and $\gamma$ under which a particular mode is isolated on a unit square domain for the Gierer-Meinhardt, Thomas and Schnakenberg models

\begin{tabular}{|c|c|c|c|c|c|c|}
\hline \multirow[t]{2}{*}{ Mode $(m, n)$} & \multicolumn{2}{|c|}{ Gierer-Meinhardt } & \multicolumn{2}{|l|}{ Thomas } & \multicolumn{2}{|c|}{ Schnakenberg } \\
\hline & $d$ & $\gamma$ & $d$ & $\gamma$ & $d$ & $\gamma$ \\
\hline$(1,0)$ & 520.1573 & 67 & 427.0152 & 44 & 10 & 29 \\
\hline$(1,1)$ & 105.1573 & 168.8 & 39.0152 & 79 & 11.5776 & 70.6 \\
\hline$(2,0)$ & 84.1573 & 238.26 & 54.0152 & 122 & 10 & 114 \\
\hline$(2,1)$ & 77.6473 & 411.42 & 29.0152 & 170 & 9.1676 & 176.72 \\
\hline$(2,2)$ & 75.1573 & 483.43 & 30.9152 & 252 & 8.6676 & 230.82 \\
\hline$(3,0)$ & 70.8473 & 619.45 & 36.0152 & 269 & 8.6176 & 265.22 \\
\hline$(3,1)$ & 72.1573 & 756.23 & 27.5152 & 320 & 8.6676 & 329.20 \\
\hline$(3,2)$ & 73.1573 & 901.38 & 28.4152 & 402 & 8.8676 & 379.21 \\
\hline$(3,3)$ & 70.5573 & 1266.99 & 28.5152 & 553 & 8.6076 & 535.09 \\
\hline$(4,0)$ & 71.1573 & 1033.51 & 31.5152 & 473 & 8.6676 & 435.99 \\
\hline$(4,1)$ & 70.3573 & 1164.86 & 27.0252 & 506 & 8.5876 & 492.28 \\
\hline$(4,2)$ & 71.1573 & 1519.87 & 27.0252 & 596 & 8.7176 & 625.35 \\
\hline$(4,3)$ & 71.1573 & 1580.66 & 27.0252 & 745 & 8.6676 & 666.82 \\
\hline$(4,4)$ & 73.1473 & 2167 & 27.0252 & 953 & 8.6076 & 909.66 \\
\hline
\end{tabular}

$$
k_{\min }^{2}=\min \left\{\forall k_{p, q}^{2}: k_{p, q}^{2}>k_{m, n}^{2} \text { for some } p, q\right\} .
$$

We impose the lower $k_{\max }^{2}$ and upper $k_{\min }^{2}$ bounds on $k_{-}^{2}$ and $k_{+}^{2}$ respectively so that $k_{m, n}^{2}$ will be the only eigenvalue with a positive real part. We wish to isolate a certain mode, that is, we want linear stability analysis to predict that the uniform steady state goes unstable only to spatial perturbations $\cos m \pi x \cos n \pi y$ with a particular $(m, n)$. Table 2 shows the parameter values $d$ and $\gamma$ for some fixed parameter values:

1. Gierer-Meinhardt model: $a=0.1, b=1.0, k=0.5$;

2. Thomas model: $a=150, b=100, K=0.05, \alpha=1.5 \rho=13$;

3. Schnakenberg model: $a=0.1$ and $b=0.9$.

\section{Appendix B. Cubic spline interpolation}

The basic idea of a cubic spline interpolation is that given a tabulated function, $x_{i}=x_{i}\left(t_{i}\right), y_{i}=y_{i}\left(t_{i}\right)$, $i=1, \ldots, n$, we determine the intermediate values of $(x, y)$ of the function say between frame 1 and 2 , that is at time 12 and $18 \mathrm{~h}$, every $15 \mathrm{~s} . n$ is the number of frames. For illustrative purposes, let us consider the time interval $t_{i}$ and $t_{i+1}, i=1, \ldots, n-1$. The aim is to obtain an interpolation formula that is smooth in the first derivative and continuous in the second derivative, both within an interval and at its boundaries. The interpolation can be shown to be of the form

$$
(x, y)=C_{0}\left(x_{j}, y_{j}\right)+C_{1}\left(x_{j+1}, y_{j+1}\right)+C_{2}\left(x_{j}^{\prime \prime}, y_{j}^{\prime \prime}\right)+C_{3}\left(x_{j+1}^{\prime \prime}, y_{j+1}^{\prime \prime}\right)
$$

with $j=1, \ldots, n b r y$, nbry is the number of boundary grid coordinates. " denotes the second derivative with respect to time, and

$$
C_{0}=\frac{t_{j+1}-t}{t_{j+1}-t_{j}}, \quad C_{1}=1-C_{0}=\frac{t-t_{j}}{t_{j+1}-t_{j}}, \quad C_{2}=\frac{1}{6}\left(C_{0}^{3}-C_{0}\right)\left(t_{j+1}-t_{j}\right)^{2}, \quad C_{3}=\frac{1}{6}\left(C_{0}^{3}-C_{0}\right)\left(t_{j+1}-t_{j}\right)^{2} .
$$

Now, the assumption of continuity of the first derivative across the boundary between any two intervals, gives rise to the system of equations 


$$
\begin{aligned}
& \frac{t_{j}-t_{j-1}}{6}\left(x_{j-1}^{\prime \prime}, y_{j-1}^{\prime \prime}\right)+\frac{t_{j+1}-t_{j-1}}{3}\left(x_{j}^{\prime \prime}, y_{j}^{\prime \prime}\right)+\frac{t_{j+1}-t_{j}}{6}\left(x_{j+1}^{\prime \prime}, y_{j+1}^{\prime \prime}\right) \\
& \quad=\frac{\left(x_{j+1}, y_{j+1}\right)-\left(x_{j}, y_{j}\right)}{t_{j+1}-t_{j}}-\frac{\left(x_{j}, y_{j}\right)-\left(x_{j-1}, y_{j-1}\right)}{t_{j}-t_{j-1}} .
\end{aligned}
$$

In our case uniqueness is guaranteed by setting both the second derivatives of $(x, y)$ at the end points equal to zero, giving the so-called natural cubic spline. Eq. (B.2) is tridiagonal, which is a huge practical advantage. Therefore the system of equations are solved in $\mathrm{O}(N)$ operations by the tridiagonal algorithm.

Once the $(\mathbf{X}, \mathbf{Y})$ boundary coordinates are obtained through the cubic spline interpolation, we apply the spring analogy to calculate and move the new interior coordinates from the old coordinates.

\section{B.1. Mesh movement using the spring analogy}

The spring analogy is a common technique of deforming a mesh [10]. It consists of replacing the mesh by fictitious springs. There are two types of springs, the vertex and the segment spring analogies. The vertex spring is used mainly to refine a particular mesh, while the segment spring is appropriate for deforming and moving a given mesh. In both cases, the segments are considered as springs. The springs are linear and Hooke's law is applied to determine the displacements of the nodes. For the segment springs, the equilibrium lengths of the springs are equal to the initial lengths of the segments. The force at each node $i$, say, is determined by

$$
\mathbf{F}_{i}=\sum_{j=1}^{n_{i}} \alpha_{i j}\left(\delta_{j}-\delta_{i}\right) .
$$

Here, $\delta_{i}$ is the displacement of node $i, n_{i}$ is the total number of nodes surrounding node $i . \alpha_{i j}$ is the spring stiffness which according to Batima [9], is given by $\alpha_{i j}=1 / \sqrt{\left(x_{i}-x_{j}\right)^{2}+\left(y_{i}-y_{j}\right)^{2}}$. At static equilibrium, $\mathbf{F}_{i}$ is zero, and applying Dirichlet boundary conditions, we obtain a system of linear algebraic equations whose unknowns are new displacements. The nodal displacements are therefore calculated as $\mathbf{x}_{i}^{\text {new }}=\mathbf{x}_{i}^{\text {old }}+\delta_{i}^{\text {final }}$.

Observe that the system

$$
\mathbf{O}_{i}=\sum_{j=1}^{n_{i}} \alpha_{i j}\left(\delta_{j}-\delta_{i}\right)
$$

is symmetric and positive definite. The resultant matrix on the left-hand side for the unknown displacements is an $M$-matrix. When a boundary is moved or deformed, the boundary nodal positions are strongly imposed by Dirichlet boundary conditions. The system is solved by a preconditioned Conjugate Gradient method where the preconditioner is determined by an incomplete Cholesky factorisation [39].

\section{References}

[1] R.A. Adams, Sobolev Spaces, Academic Press, New York, 1975.

[2] J.L. Aragón, C. Varea, R.A. Barrio, P.K. Maini, Spatial patterning in modified turing systems: application to pigmentation patterns on Marine fish, Forma 13 (1998) 213-221.

[3] J.L. Aragón, M. Torres, D. Gil, R.A. Barrio, P.K. Maini, Turing patterns with pentagonal symmetry, Phys. Rev. E 65 (2002) 1-9.

[4] M.J. Baines, Moving finite elements, Monographs on Numerical Analysis, Clarendon Press, Oxford, 1994.

[5] M.J. Baines, A.J. Wathen, Moving finite element methods for evolutionary problems. I. Theory, J. Comp. Phys. 79 (1988) 245269.

[6] Y.I. Balkarei, A.V. GrigörYants, Y.A. Rhzanov, M.I. Elinson, Regenerative oscillations, spatial-temporal single pulses and static inhomogeneous structures in optically bistable semiconductors, Opt. Commun. 66 (1988) 161-166. 
[7] R.A. Barrio, C. Varea, J.L. Aragón, P.K. Maini, A two-dimensional numerical study of spatial pattern formation in interacting systems, Bull. Math. Biol. 61 (1999) 483-505.

[8] R.A. Barrio, P.K. Maini, J.L. Aragón, M. Torres, Size-dependent symmetry breaking in models for morphogenisis, Physica D 2920 (2002) 1-12.

[9] J.T. Batima, Unsteady Euler airfoil solutions using unstructured dynamic meshes, AIAA J. 28 (8) (1990) $1381-1388$.

[10] J.F. Blom, Considerations of the spring analogy, Int. J. Numer. Meth. Fluids 12 (2000) 647-668.

[11] S.C. Brenner, L.R. Scott, The Mathematical Theory of Finite Element Methods, Springer, Berlin, 1994.

[12] N.N. Carlson, K. Miller, Design and application of a gradient-weighted moving finite element code I: in one dimension, SIAM J. Sci. Comput. 19 (3) (1998) 728-765.

[13] N.N. Carlson, K. Miller, Design and application of a gradient-weighted moving finite element code II: in two dimensions, SIAM J. Sci. Comput. 19 (3) (1998) 766-798.

[14] E.J. Crampin, Reaction-diffusion patterns on growing domains, D. Phil. Thesis, University of Oxford, 2000.

[15] E.J. Crampin, E.A. Gaffney, P.K. Maini, Reaction and diffusion on growing domains: scenarios for robust pattern formation, Bull. Math. Biol. 61 (2002) 1093-1120.

[16] L. Edelstein-Keshet, Mathematical Models in Biology, Random House, New York, 1988.

[17] P. Gray, S.K. Scott, Autocatalytic reactions in the isothermal, continuous stirred tank reactor: isolas and other forms of multistability, Chem. Eng. Sci. 38 (1) (1983) 29-43.

[18] P. Gray, S.K. Scott, Autocatalytic reactions in the isothermal, continuous stirred tank reactor: oscillations and the instabilities in the system $A+2 B \rightarrow 3 B, B \rightarrow C$, Chem. Eng. Sci. 39 (6) (1984) 1087-1097.

[19] A. Gierer, H. Meinhardt, A theory of biological pattern formation, Kybernetik 12 (1972) 30-39.

[20] P.K. Jimack, A.J. Wathen, Temporal derivatives in the finite element method on continuously deforming grids, SIAM J. Numer. Anal. 28 (4) (1991) 990-1003.

[21] C. Johnson, Numerical Solution of Partial Differential Equations by the Finite Element Method, Cambridge University Press, Cambridge, MA, 1987.

[22] S. Kondo, R. Asai, A reaction-diffusion wave on the skin of the marine angelfish Pomacanthus, Nature 376 (1995) $765-768$.

[23] V.I. Krinsky, Self-organisation, Auto-Waves and Structures Far From Equilibrium, Springer, Berlin, 1984.

[24] A. Madzvamuse, A numerical approach to the study of spatial pattern formation, D. Phil. Thesis, University of Oxford, 2000.

[25] A. Madzvamuse, P.K. Maini, A.J. Wathen, T. Sekimura, A predictive model for color pattern formation in the butterfly wing of Papilio dardanus, Hiroshima Math. J. 32 (2002) 325-336.

[26] A. Madzvamuse, R.D.K. Thomas, P.K. Maini, A.J. Wathen, A numerical approach to the study of spatial pattern formation in the Ligaments of Arcoid Bivalves, Bull. Math. Biol. 64 (2002) 501-530.

[27] P.K. Maini, M. Solursh, Cellular mechanisms of pattern formation in the development of limb, Int. Rev. Cytol. 129 (1991) 91-133.

[28] H. Meinhardt, The Algorithmic Beauty of Sea Shells, Springer, Heidelberg, New York, 1995.

[29] K. Miller, R.N. Miller, Moving finite elements. Part I, SIAM J. Numer. Anal. 18 (1981) 1019-1032.

[30] K. Miller, Moving finite elements. Part II, SIAM J. Numer. Anal. 18 (1981) 1033-1057.

[31] J.D. Müller, P.L. Roe, H. Deconinck, A frontal approach for internal node generation for Delaunay triangulation, Int. J. Numer. Methods Fluids 17 (3) (1993) 241-256.

[32] J.D. Murray, On pattern formation mechanisms for lepidopteran wing patterns and mammalian coat markings, Philos. Trans. Roy. Soc. London B 295 (1981) 473-496.

[33] J.D. Murray, Mathematical Biology, Springer, Heidelberg, New York, 1993.

[34] H.F. Nijhout, A comprehensive model for colour pattern formation in butterflies, Proc. Roy. Soc. London B 239 (1990) 81-113.

[35] T. Nozakura, S. Ikeuchi, Formation of dissipative structures in galaxies, Astrophys. J. 279 (1984) 40-52.

[36] K.J. Painter, Chemotaxis as a mechanism for morhogenesis, D. Phil. Thesis, University of Oxford, 1997.

[37] K.J. Painter, P.K. Maini, H.G. Othmer, Stripe formation in juvenile Pomacanthus explained by a generalised Turing mechanism with chemotaxis, Proc. Natl. Acad. Sci. USA 96 (1999) 5549-5554.

[38] T.N. Reddy, An Introduction to the Finite Element Method, McGraw-Hill, New York, 1984.

[39] Y. Saad, SPARSEKIT, a basic tool kit for sparse matrix computations, 1994, http://www-users.cs.umn.edu/ saad/.

[40] Y. Saad, Iterative Methods for Sparse Linear Systems, PWS Publishing, 1996.

[41] J. Schnakenberg, Simple chemical reaction systems with limit cycle behavior, J. Theoret. Biol. 81 (1979) 389-400.

[42] L.A. Segel, J.L. Jackson, Dissipative structure: an explanation and an ecological example, J. Theoret. Biol. 37 (1972) 545559.

[43] T. Sekimura, P.K. Maini, J.B. Nardi, M. Zhu, J.D. Murray, Pattern formation in lepidopteran wings, Comm. Theoret. Biol. 5 (24) (1998) 69-87.

[44] T. Sekimura, M. Zhu, J. Cook, P.K. Maini, J.D. Murray, Pattern formation of scale cells in lepidoptera by differential origindependent cell adhesion, Bull. Math. Biol. 61 (1999) 807-827.

[45] T. Sekimura, A. Madzvamuse, A.J. Wathen, P.K. Maini, A model for colour pattern formation in the butterfly wing of Papilio dardanus, Proc. Roy. Soc. London B 267 (2000) 851-859. 
[46] W. Sun, T. Tang, J.M. Ward, J. Wei, Numerical challenges for resolving spike dynamics for two reaction-diffusion systems, Stud. Appl. Math. III (2003) 41-84.

[47] D. Thomas, Artificial enzyme membrane, transport, memory and oscillatory phenomena, in: D. Thomas, J.-P. Kervenez (Eds.), Analysis and Control of Immobilised Enzyme Systems, Springer, Berlin, Heidelberg, New York, 1975, pp. 115-150.

[48] R.D.K. Thomas, A. Madzvamuse, P.K. Maini, A.J. Wathen, Growth patterns of noetiid ligaments: implications of developmental models for the origin of an evolutionary novelty among arcoid bivalves, in: E.M. Harper, J.D. Taylor, J.A. Crame (Eds.), The Evolutionary Biology of the Bivalvia, vol. 177, Geological Society Special Publication, 2000, pp. 279-289.

[49] A.M. Turing, The chemical basis of morphogenesis, Philos. Trans. Roy. Soc. London B 237 (1952) 37-72.

[50] D.B. White, The planforms and onset of convection with a temperature dependent viscosity, J. Fluid Mech. 191 (1988) $247-286$. 